\title{
Modeling conformationally flexible proteins with X-ray scattering and molecular simulations
}

\author{
Melissa S. Gildenberg ${ }^{a}$ and M. Todd Washington ${ }^{b}$ \\ aUniversity of lowa, 51 Netwon Rd., lowa City, IA 52245, melissa-gildenberg@uiowa.edu \\ bUniversity of lowa, 51 Netwon Rd., lowa City, IA 52245, todd-washington@uiowa.edu \\ Conformationally flexible proteins play pivotal roles in various cellular processes, \\ including DNA replication and repair, cell cycle regulation, and signal transduction. \\ Historically it has been difficult to obtain meaningful structural information about these \\ molecules due to their disordered nature. However, recent advancements in hybrid \\ approaches that pair low-resolution structural techniques with computational methods \\ have allowed us to better understand the structure and function of flexible proteins. \\ Proteins that sample only a limited number of conformational states may be described \\ by approaches that combine small angle X-ray scattering (SAXS) and minimal ensemble \\ searches (MES). However, more complex proteins require broader characterization of \\ their full range of flexibility. We have combined SAXS with full ensemble Brownian \\ Dynamics (BD) simulations to better understand the role of intrinsic disorder in protein \\ function. We have shown this technique to be valuable in the study of proteins with \\ flexible linkers connecting folded domains, proteins with long intrinsically disordered \\ tails, and proteins for which limited structural information is currently available. Moving \\ forward, full ensemble hybrid techniques can be applied to many more conformationally \\ flexible proteins to provide vital structural information about these elusive systems.
}

